\title{
Conformable Fractional Cosine Families of Operators
}

\author{
M. Elomari ${ }^{1 *}$, S. Melliani ${ }^{1}$ and L. S. Chadli ${ }^{1}$ \\ ${ }^{1}$ Laboratory of Applied Mathematics and Scientific Calculus, Sultan Moulay Slimane University, BP 523, 23000, Beni Mellal, Morocco \\ * Corresponding author
}

\author{
Article Info \\ Keywords: $\alpha$-cosine families, Con- \\ formable derivative, Mild solution. \\ 2010 AMS: 26433 \\ Received: 21 June 2018 \\ Accepted: 21 January 2019 \\ Available online: August 2019
}

\begin{abstract}
In this paper we are concerned with the problem

$$
\begin{gathered}
\left\{\begin{array}{l}
u^{(\alpha)}(t)=A u(t)+f(t, u(t)) \\
u(0)=u_{0}, D^{\alpha} u(0)=u_{1}
\end{array} \quad t \in[0, T]\right. \\
\left\{\begin{array}{l}
u^{(\alpha)}(t)=A u(t)+f(t, u(t)) \\
u(0)=u_{0}, D^{\alpha} u(0)=u_{1}
\end{array}\right.
\end{gathered}
$$
\end{abstract}

Where $\alpha \in(1,2]$, and we use the conformable derivative. We give the notion of $\alpha$-Cosine families and proveded the existence and uniqueness of the problem 0.1 .

\section{Introduction}

Our primary objective is to investigate the abstract semi-linear $\alpha$-order initial value problem

$$
\begin{gathered}
u^{(\alpha)}(t)=A u(t)+f(t, u(t)) \quad t \in[0, T] \\
u(0)=u_{0}, D^{\alpha} u(0)=u_{1}
\end{gathered}
$$

Where $T \in \mathbb{R}_{+}^{*}, 1<\alpha<2$, the linear operator $A: D(A) \subset X \rightarrow X, f:[0, T] \times X \rightarrow X, \mathscr{C}=\mathscr{C}([0, T], X)$ is the Banach space of all continuous functions equipped with the supnorm.

A is the (possibly unbounded) infinitesimal generator of a strongly continuous $\alpha$-cosine family of linear operators in $X$ and fis a nonlinear mapping from $f:[0, T] \times X \rightarrow X$. Our goal will be to give a systematic and general treatment of (1) from the standpoint of existence, uniqueness of solutions.

The pioneering work concerning the new definition of fractional derivative was done by [6]. This work open the door in front of sevral works in this sens. Thabet Abdeljawad and other authors feat this notion for introducing the basic definition of $\alpha$-semigroup. But G. F. WEBB in [4] annonces the way to give an integral solution for one second problem, They precisely have considered the notion of cosine families. In this paper we will try to give the definition of $\alpha$-cosine families, and provide the existence and uniqueness of the problem (1).

This paper is organised as follows. In section 2 we recall some notion concerning the new derivative. Section 3 deal with the Basic definition of $\alpha$-Cosine families and some properties. The proof of mains results takes place in the last Section.

\section{Preliminaries}

In this section we will give some definition and properties concerning the new derevative important in the following.

Definition 2.1. see[6] Let $\alpha \in(n, n+1]$ and $f:[0, \infty) \rightarrow \mathbb{R}$ be $n$-differentiable at $t>0$, then the conformable fractional derivative of $f$ of order $\alpha$ is defined by

$$
\begin{aligned}
& f^{(\alpha)}(t)= \lim _{\varepsilon \rightarrow 0} \frac{f^{(n)}\left(t+\varepsilon t^{n+1-\alpha}\right)-f^{(n)}(t)}{\varepsilon} \\
& f^{(\alpha)}(0)=\lim _{t \rightarrow 0} f^{(\alpha)}(t)
\end{aligned}
$$


Remark 2.2. see[6] As consequence of the previous definition, one can easily show that

$$
f^{(\alpha)}(t)=t^{n+1-\alpha} f^{(n+1)}(t)
$$

where $\alpha \in(n, n+1]$, and $f$ is $(n+1)$-differentiable at $t>0$.

Definition 2.3. see[6] Let $\alpha \in(1,2],\left(I^{\alpha} f\right)(t)=\int_{0}^{t} s^{\alpha-2} f(s) d s$.

Theorem 2.4. see[6]

$$
\left(I^{\alpha} f\right)^{(\alpha)}(t)=f(t)
$$

for $t \geq 0$

Example 2.5.

where $\alpha \in(1,2)$

$$
I^{\alpha}(\sin (t))=\sum_{n=0}^{\infty} \frac{(-1)^{n} t^{2 n+\alpha}}{(2 n+\alpha)(2 n+1) !}
$$

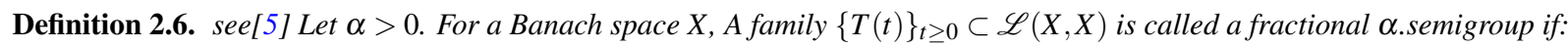
1. $T(0)=I$
2. $T\left((s+t)^{\frac{1}{\alpha}}\right)=T\left(s^{\frac{1}{\alpha}}\right) T\left(t^{\frac{1}{\alpha}}\right)$, for all $s, t \in[0, \infty)$

Example 2.7. Let $A$ be a bounded linear operator on $X$. Define $T(t)=e^{2 \sqrt{t} A}$. Then $T(t)_{t \geq 0}$ is a $\frac{1}{2}$ semigroup. Indeed:

1. $T(0)=e^{0 A}=I$

2. $\forall s, t \in[0, \infty), T\left((s+t)^{2}\right)=e^{2(t+s) A}=e^{2 t A} e^{2 s A}=T(s) T(t)$

Definition 2.8. see[5] An $\alpha$-semigroup $T(t)$ is called a $c_{0}$-semigroup if, for each fixed $x \in X, T(t) x \rightarrow x$ as $t \rightarrow 0^{+}$

The conformable $\alpha$-derivative of $T(t)$ at $t=0$ is called the $\alpha$-infinitesimal generator of the fractional $\alpha$-semigroup $T(t)$, with domain equals

$$
\left\{x \in X, \lim _{t \rightarrow 0} T(t) x \text { exist }\right\}
$$

\section{3. $\alpha$-Cosine families}

We will give the following definition

Definition 3.1. A one parameter family $C^{\alpha}(t), t \in \mathbb{R}$ of bounded linear operators mapping the Banach space $X$ into itself is called a strongly continuous $\alpha$-cosine family if and only if

1. $C^{\alpha}(0)=I$

2. $C^{\alpha}\left((s+t)^{\frac{1}{\alpha}}\right)+C^{\alpha}\left((s-t)^{\frac{1}{\alpha}}\right)=2 C^{\alpha}\left(s^{\frac{1}{\alpha}}\right) C^{\alpha}\left(t^{\frac{1}{\alpha}}\right)$

3. The mapping $t \rightarrow C^{\alpha}(t) x$ is a continuous mapping for each fixed $x \in X$.

If $C^{\alpha}(t), t \in \mathbb{R}$ is a strongly continuous $\alpha$-cosine family in $X$, then: $S^{\alpha}(t), t \in \mathbb{R}$ is the one parameter family of operators in $X$ defined by

$$
S^{\alpha}(t)=\left(I^{\alpha} S^{\alpha}\right)(t)
$$

Example 3.2. Let $A$ be a bounded linear operator on $X$. Define $C^{\alpha}(t)=\frac{e^{2 \sqrt{t A}}+e^{-2 \sqrt{t} A}}{2}$. Then $T(t)_{t \geq 0}$ is a $\frac{1}{2}$ semigroup. Indeed:

1. $C^{\alpha}(0)=e^{0 A}=I$

2. $C^{\alpha}\left((t+s)^{2}\right)+C^{\alpha}\left((t-s)^{2}\right)=\frac{e^{2(t+s) A}+e^{-2(t+s) A}}{2}+\frac{e^{2(t-s) A}+e^{-2(t-s) A}}{2}=2 C^{\alpha}\left(t^{2}\right) C^{\alpha}\left(s^{2}\right)$

3. The continuity is clear

Proposition 3.3. The family $\left\{C^{\alpha}(t), t \in R\right\}$ is a strongly cosine families if only if $\left\{C(t)=C^{\alpha}(t)\left(t^{\frac{1}{\alpha}}\right), t \in R\right\}$ is a strongly continuous cosine families

Proof. Necessary condition: It is clear that $C(0)=I$. For all $s, t \in \mathbb{R}$, we have

$$
\begin{gathered}
C(t+s)+C(t-s)=C^{\alpha}(t+s)\left(t^{\frac{1}{\alpha}}\right)+C^{\alpha}(t-s)\left(t^{\frac{1}{\alpha}}\right) \\
=2 C^{\alpha}(t)\left(t^{\frac{1}{\alpha}}\right) C^{\alpha}(s)\left(t^{\frac{1}{\alpha}}\right) \\
=2 C(t) C(s)
\end{gathered}
$$

Further the continuity of $t \rightarrow C_{q}^{\alpha}\left(t^{\frac{1}{\alpha}}\right) x$ and the continuity of $t \rightarrow t^{\alpha}$ implies that $t \rightarrow C(t) x$ is continuous.

For the sufficient condition it suffice to note that $C t^{\alpha}=C^{\alpha}(t)$

If $\left\{C^{\alpha}(t), t \in \mathbb{R}\right\}$ is a strongly continuous cosine family in $X$, then $\left\{S^{\alpha}(t), t \in \mathbb{R}\right\}$ is the one parameter family of operators in $X$ defined by

$$
S^{\alpha}(t) x=\left(I C^{\alpha}\right)(t) x, \forall t \in \mathbb{R}, x \in X
$$

Remark 3.4. As the previous proposition $\left\{S^{\alpha}(t), t \in \mathbb{R}\right\}$ is a $\alpha$-Sine family iff $\left\{S(t)=S^{\alpha}\left(t^{\frac{1}{\alpha}}\right), t \in \mathbb{R}\right\}$ is sine family. 
Proposition 3.5. Let $\left.C^{\alpha}(t), t \in \mathbb{R}\right\}$ be a strongly continuous cosine family in $X$.

The following are true:

1. $C^{\alpha}(t)=C^{\alpha}(-t) \forall t \in \mathbb{R}$

2. $C^{\alpha}(s), S^{\alpha}(s), C^{\alpha}(t)$, and $S^{\alpha}(t)$ commute for all $s, t \in \mathbb{R}$

3. $S^{\alpha}(t) x$ is continuous in $t$ on $\mathbb{R}$ for each fixed $x \in X$

4. $S^{\alpha}(s+t)+S^{\alpha}(s-t)=2 S^{\alpha}(s) C^{\alpha}(t)$ for all $s, t \in \mathbb{R}$

5. $S^{\alpha}(s+t)=S^{\alpha}(s) C^{\alpha}(t)+S^{\alpha}(t) C^{\alpha}(s)$ for all $s, t \in \mathbb{R}$

6. $S^{\alpha}(t)=-S^{\alpha}(-t)$ for all $t \in \mathbb{R}$

7. There exist constants $M>1$ and $\omega \geq 0$ such that $C^{\alpha}(t) \leq M e^{\omega t^{\alpha}}$ for all $t \in \mathbb{R}$ and

$$
\left\|S^{\alpha}\left(t_{1}\right)-S^{\alpha}\left(t_{2}\right)\right\| \leq \frac{M}{\omega}\left(e^{\omega t_{1}^{\alpha}}-e^{\omega t_{2}^{\alpha}}\right)
$$

Proof. The proposition 1-6 are consequence of the proposition 3.1. For 7, we have

$$
\begin{gathered}
\left\|S^{\alpha}\left(t_{1}\right)-S^{\alpha}\left(t_{2}\right)\right\|=\int_{t_{2}}^{t_{1}} \frac{C^{\alpha}(s)}{s^{1-\alpha}} d s \\
\leq M \int_{t_{2}}^{t_{1}} \frac{e^{\omega s^{\alpha}}}{s^{1-\alpha}} d s=\left[e^{\omega s}\right]_{t_{2}}^{t_{1}}
\end{gathered}
$$

The $\alpha$-infinitesimal generator of a strongly continuous $\alpha$-cosine families $C^{\alpha}(t), t \in \mathbb{R}$ is the operator $A: X \rightarrow X$ defined by

$$
\begin{gathered}
A x=\lim _{t \rightarrow 0} D^{\alpha} C^{\alpha}(t) \\
D(A)=\left\{x, t \rightarrow D^{\alpha} C^{\alpha}(t) x, \text { is continuous of } t\right\} \\
E=\left\{x, t \rightarrow D C^{\alpha}(t) x, \text { is continuous of } t\right\}
\end{gathered}
$$

Lemma 3.6.

$$
C(t)=\lim _{\alpha \rightarrow 1^{+}} C^{\alpha}(t) \text { is a cosine families }
$$

Proof. It suffice to note that $C^{\alpha}\left(t^{\frac{1}{\alpha}}\right)$ is a cosine families, $t \rightarrow t^{\alpha}$ is continuous.

Proposition 3.7. Let $C^{\alpha}(t), t \in \mathbb{R}$, be a strongly continuous $\alpha$-cosine family in $X$ with $\alpha$-infinitesimal generator $A$. The following are true.

1. $D(A)$ is dense in $X$ and $A$ is a closed operator in $X$.

2. if $x \in X$ and $r, s \in \mathbb{R}$, then $z=\int_{r}^{s} \frac{S^{\alpha}(u)}{u^{1-\alpha}} x d u \in D(A)$ and $A z=C^{\alpha}(s) x-C^{\alpha}(r) x$

3. if $x \in X$ and $r, s \in \mathbb{R}$, then $z=\int_{0}^{s} \int_{0}^{r} \frac{C^{\alpha}(u)}{u^{1-\alpha}} \frac{C^{\alpha}(v)}{v^{1-\alpha}} d v d u x \in D(A)$ and $A z=2^{-1}\left(C^{\alpha}(s+r) x-C^{\alpha}(s-r) x\right)$

4. if $x \in X$, then $S^{\alpha}(t) x \in E$

5. if $x \in E$, then $S^{\alpha}(t) x \in D(A)$ and $\left(C^{\alpha}\right)^{(\alpha)}(t) x=A S^{\alpha}(t) x$

6. if $x \in D(A)$, then $C^{\alpha}(t) x \in D(A)$ and $D^{\alpha} C^{\alpha}(t) x=A C^{\alpha}(t) x=C^{\alpha}(t) A x$

7. if $x \in E$, then $\lim _{t \rightarrow 0} A S^{\alpha}(t) x=0$

8. if $x \in E$, then $S^{\alpha}(t) x \in D(A)$ and $D^{\alpha} S^{\alpha}(t) x=A S^{\alpha}(t) x$

9. if $x \in D(A)$, then $S^{\alpha}(t) x \in D(A)$ and $A S^{\alpha}(t) x=S^{\alpha}(t) A x$

10. $C^{\alpha}(t+s)-C^{\alpha}(t-s)=2 A S^{\alpha}(t) S^{\alpha}(s)$ for all $s, t \in \mathbb{R}$.

Proof. Use the previous lemma.

For 1 it suffuce to use the previous lemma and proposition 2.2 in [4].

For $2-10$ By change $s$ by $s^{\frac{1}{\alpha}}$ and $t$ by $t^{\frac{1}{\alpha}}$ and use proposition 2.2 in [4].

\section{Mains results}

In this section we consider the problem

$$
\begin{gathered}
u^{(\alpha)}(t)=A u(t)+f(t, u(t)) \quad t>0, \alpha \in(1,2) \\
u(0)=u_{0}, u^{(\alpha)}(0)=u_{1}
\end{gathered}
$$

Where $A: D(A) \subset X \rightarrow X$ is a linear operator $\alpha$ infinitisimal generator of a $C^{\alpha}(t), t \in \mathbb{R}$-Cosine families, and $u_{0} \in X$. We set $\mathscr{C}=\mathscr{C}([0, T], X)$. $f:[0, T] \times X \rightarrow X$ is continuous and satisfies a Lipschitz condition $\left\|f(x, y)-f\left(x, y^{\prime}\right)\right\| \leq M_{f}\left\|y-y^{\prime}\right\|, \forall x \in[0, T], x, y \in X$. $\mathrm{T}$ he folowing defiition is a extension of usual definition of mild see [4].

Definition 4.1. A function $u:[0, \infty) \rightarrow X$ is a mild solution of (1) if
1. $u$ is continuous differential on $[0, \infty)$
2. $u$ is continuously $\alpha$-differentiable on $(0, \infty)$
3. $u(t) \in D(A)$ for $t>0$,
4. $u(t)=C^{\alpha}(t) u_{0}+S^{\alpha}(t) u_{1}+\int_{0}^{t} \frac{S^{\alpha}(t-s) f(s, u(s))}{s^{2-\alpha}} d s$ 
Theorem 4.2. Suppose that $f:[0, T] \times X \rightarrow X$ is continuous and Lipschitzian with respect to the second argument, then for any $u_{0}, u_{1} \in X$ such that $C^{\alpha}(t) u_{0}, S^{\alpha} u_{1} \in D(A)$ for all $t>0$, the problem (1) has a unique mild solution

Proof. Define the operator $P: \mathscr{C} \rightarrow \mathscr{C}$ By

$$
(P u)(t)=C^{\alpha} u_{0}+S^{\alpha} u_{1}+\int_{0}^{t} s^{\alpha-2} S^{\alpha}(t-s) f(s, u(s))
$$

We give the proof in sevral step:

Step 1: Let $u_{1}, u_{2} \in \mathscr{C}$

$$
\begin{aligned}
& |(P u)(t+h)-(P u)(t)|=\left|\int_{0}^{t+h} s^{\alpha-1} S^{\alpha}(t+h-s) f(s, u(s)) d s-\int_{0}^{t} s^{\alpha-2} S^{\alpha}(t-s) f(s, u(s)) d s\right| \\
& \leq\left|\int_{0}^{t+h} s^{\alpha-1}\left(S^{\alpha}(t+h-s)-S^{\alpha}(t-s)\right) f(s, u(s)) d s\right|+\left|\int_{0}^{t} s^{\alpha-2} S^{\alpha}(t+h-s) f(s, u(s)) d s\right|
\end{aligned}
$$

But

$$
\left|s^{\alpha-1}\left(S^{\alpha}(t+h-s)-S^{\alpha}(t-s)\right) f(s, u(s))\right| \leq T^{\alpha-1} M h e^{\omega T^{\alpha}} \sup _{s \in[0, T]}\|f(s, u(s))\|
$$

By the dominated convergence theorem

$$
\int_{0}^{t+h} s^{\alpha-2}\left(S^{\alpha}(t+h-s)-S^{\alpha}(t-s)\right) f(s, u(s)) d s \rightarrow 0, \text { as } h \rightarrow 0
$$

Also

$$
\left|s^{\alpha-1} S^{\alpha}(t+h-s) f(s, u(s))\right| \leq T^{\alpha-2} M e^{\omega h} \sup _{s \in[0, T]}\|f(s, u(s))\|
$$

By the dominated convergence theorem

$$
\left|\int_{0}^{t} s^{\alpha-2} S^{\alpha}(t+h-s) f(s, u(s)) d s\right| \rightarrow 0, \text { as } h \rightarrow 0
$$

Hence $P u \in \mathscr{C}$ i.e $P$ maps $\mathscr{C}$ into itself.

Step 2: Let $u_{1}, u_{2} \in \mathscr{C}, t \in[0, T]$, with $u_{1}(0)=u_{2}(0)=u_{0}$

$$
\begin{gathered}
\left\|\left(P u_{1}\right)(t)-\left(P u_{2}\right)(t)\right\| \leq\left\|C^{\alpha}(t)\left(u_{1}(0)-u_{2}(0)\right)\right\|+\left\|S^{\alpha}(t)\left(u_{1}(0)-u_{2}(0)\right)\right\|+\left\|s^{\alpha-2} S^{\alpha}(t-s)\left(f\left(s, u_{1}(s)\right)-f\left(s, u_{2}(s)\right)\right)\right\| \\
\leq M M_{f} e^{\omega T^{\alpha}}\left\|u_{1}-u_{2}\right\| \int_{0}^{t} s^{\alpha-1} d s \\
\leq t^{\alpha} \frac{M M_{f} e^{\omega T^{\alpha}}}{2}
\end{gathered}
$$

We set $\eta=\frac{M M_{f} e^{\omega T^{\alpha}}}{2}$ We can deduce that

$$
\begin{aligned}
\|\left(P^{2} u_{1}\right)(t)- & \left(P^{2} u_{2}\right)(t)\left\|\leq \eta \int_{0}^{t} s^{\alpha}\right\| u_{1}-u_{2} \| d s \\
& \leq \frac{\left(\eta t^{\frac{\alpha}{2}}\right)^{2}}{2}\left\|u_{1}-u_{2}\right\|
\end{aligned}
$$

And by induction, we have for all $t \in[0, T]$

$$
\left\|\left(P^{2} u_{1}\right)(t)-\left(P^{2} u_{2}\right)(t)\right\| \leq \frac{\left(\eta t^{\frac{\alpha}{2}}\right)^{n}}{n !}\left\|u_{1}-u_{2}\right\|
$$

Since $\frac{\left(\eta t^{\frac{\alpha}{2}}\right)^{n}}{n !} \rightarrow 0$, as $n \rightarrow \infty$, then there exists $r \in \mathbb{N}$ such that follows that $P^{r}$ is a contraction and there exists a unique $u \in \mathscr{C}$ such that $P^{r} u=x$. Furthermore, we have

$$
P^{r}(P u)=P\left(P^{r} u\right)=P u
$$

Hence $P u$ is a unique fixed point of $P^{r}$, so we conclude that $u$ is the unique mild solution of (1).

\section{Conclusion}

In this section you should present the conclusion of the paper. Conclusions must focus on the novelty and exceptional results you acquired. Allow a sufficient space in the article for conclusions. Do not repeat the contents of Introduction or the Abstract. Focus on the essential things of your article.

\section{Acknowledgement}

This is a text of acknowledgements. Do not forget people who have assisted you on your work. Do not exaggerate with thanks. If your work has been paid by a Grant, mention the Grant name and number here. 


\section{References}

[1] Agarwal, RP, Zhou, H, He, Y: Existence of fractional neutral functional differential equations. Comput. Math. Appl. 59(3), 1095-1100 (2010)

[2] Abdeljawad, T., On Conformable Fractional Calculus,Journal of Computational ad Applied Mathematics,Vol. 279, 1 May 2015, 57-66, arXiv: 1402.6892v1 [math D, S] 27 Feb 2014.

[3] Balakrishnan, A. V., Fractional Powers Of Closed Operators And The Semigroups Generated By Them, Pacific Journal of Mathematics 10, pp. 419-439, 1960

[4] TRAVIS. C. C and WEBB. G. F. Cosine Families and abstract nonlinear second order differential equations. Acta Mathematica Academiae Seientiarum Hungaricae Tomus 32 (3-4), (1978), 75-96.

[5] Mohammed AL Horani. Roshdi Khalil and Thabet Abdeljawad. Conformable Fractional Semigroups of Operators. arXiv:1502.06014v1 [math.FA] 21 Nov 2014 Conformable.

[6] Khalil, R., Al Horani, M., Yousef. A. and Sababheh, M., A new Definition Of Fractional Derivative, J. Comput. Appl. Math. 264. pp. 65?0, 2014.

[7] Kilbas, AA, Srivastava, HH, Trujillo, JJ: Theory and Applications of Fractional Differential Equations. Elsevier, Amsterdam (2006)

[8] Pazy, A., Semigroups of Linear Operators and Applications to Partial Differntial Equations, Springer-Verlag, 1983. 